\title{
Hydraulic falls under a floating ice plate due to submerged obstructions
}

\author{
C. Page $^{1}$ and E. I. Părău ${ }^{2, \dagger}$ \\ ${ }^{1}$ School of Computing Sciences, University of East Anglia, Norwich NR4 7TJ, UK \\ ${ }^{2}$ School of Mathematics, University of East Anglia, Norwich NR4 7TJ, UK
}

(Received 18 October 2013; revised 29 January 2014; accepted 11 February 2014; first published online 18 March 2014)

Steady two-dimensional nonlinear flexural-gravity hydraulic falls past a submerged obstruction on the bottom of a channel are considered. The fluid is assumed to be ideal and is covered above by a thin ice plate. Cosserat theory is used to model the sheet of ice as a thin elastic shell, and boundary integral equation techniques are then employed to find critical flow solutions. By utilising a second obstruction, solutions with a train of waves trapped between two obstructions are investigated.

Key words: surface gravity waves, waves/free-surface flows

\section{Introduction}

The classic hydroelastic problem concerning the interaction between a deformable body and a moving fluid is a widely studied area in mathematics and engineering, because of its vast range of physical applications (Korobkin, Părău \& Vanden-Broeck 2011). One such application involves the modelling of an ice plate floating on top of water as a thin elastic sheet (Squire et al. 1996). During the winter months, the ocean in the Arctic and Antarctic regions is frozen so that vast continuous ice plates cover the water. These ice plates are used for transportation links, in the form of aircraft runways, roads and railway lines for trains. The interaction of the deformable floating ice plate and the underlying ocean water waves is therefore of concern and can indeed be fatal. The moving load on the ice plate results in waves propagating through the fluid. There is also much interest in shallower-water applications, such as ice plates floating on rivers and lakes. Experiments with moving loads have been done at Lake Saroma in Hokkaido, Japan (see Takizawa 1985, 1988) and Lake Diefenbaker in Southern Saskatchewan, Canada (see Eyre 1977). Again, transportation links over the ice plates are of importance (for example, railways have been placed over the river $\mathrm{Ob}$ and the lake Baikal in Siberia, Russia (see Squire et al. 1996 for more details)). Air-cushioned vehicles are sometimes used to break the ice, and the resulting deformation of the ice plate is also of concern (Ashton 1986).

When the ice deformations and water waves are of small amplitude, linear theories have been used to model the ice plate (see, for example, Squire et al. 1996). However, the linear theory fails when the moving load is near critical speed. Further, Squire (2011) provides detailed insight into how global warming can have significant effects

$†$ Email address for correspondence: e.parau@uea.ac.uk 
on the Antarctic and Arctic sea-ice conditions due to, for example, warmer summers resulting in increased melting of the ice and thus rougher sea conditions. The linear theory is therefore becoming increasingly limited, and so nonlinear models have been developed.

Kirchhoff-Love elasticity models have been adopted, and periodic waves (Forbes 1986, 1988b), elevation and depression solitary waves with decaying oscillations in their tails (Părău \& Dias 2002) and generalized solitary waves (Vanden-Broeck \& Părău 2011) have been obtained. Părău \& Dias (2002) considered nonlinear effects when the load speed is near critical, in both shallow and deep water. Elevation and depression solitary waves with decaying oscillations in their tails were found for particular water-depth ranges, using a forced nonlinear Schrödinger equation. Milewski, Vanden-Broeck \& Wang (2011) obtained pure hydroelastic solitary waves for the full nonlinear model in deep water.

The Kirchhoff-Love model does not appear to have a conservative form, however, so recently Plotnikov \& Toland (2011) have considered the ice plate as a thin elastic shell and used the special Cosserat theory of hyperelastic shells, satisfying Kirchhoff's hypothesis (see Antman 2005 for more details), to model the interactions between the ice plate and an infinite ocean. Guyenne \& Părău (2012) and Wang, Vanden-Broeck \& Milewski (2013) have used this Cosserat formulation to compute pure solitary wave solutions with near-critical wave speeds in infinite depth. Guyenne \& Părău (2014) studied solitary waves in finite depth. They compared their fully nonlinear results with weakly nonlinear solutions of a forced nonlinear Schrödinger equation and a fifth-order Korteweg-de Vries (KdV) equation. A fifth-order $\mathrm{KdV}$ equation was also derived by Xia \& Shen (2002) for shallow water waves in channels covered by ice.

In this paper, we use Cosserat theory with boundary integral equation techniques to investigate flexural-gravity hydraulic falls (conjugate flows) over submerged obstructions. We therefore look for flows where the depth of the fluid under the ice plate is different upstream and downstream of the obstacle (see, for example, Dias \& Vanden-Broeck 2002). In the absence of an ice plate, when the forces of both gravity and surface tension are considered, gravity-capillary hydraulic falls have been obtained (Guayjarernpanishk \& Asavanant 2012; Page, Grandison \& Părău 2014). The flexural-gravity problem has similarities to the gravity-capillary problem, but has additional complexity in the dynamic boundary condition.

In the next section, the problem is formulated mathematically. Our results are then presented and discussed in $\S 3$, and we conclude with a summary of the results and a discussion about the physical relevance of our findings in $\S 4$.

\section{Formulation}

We consider an inviscid, incompressible fluid flowing along a channel. One or multiple submerged obstructions exist on the bottom of the channel, and the fluid is covered above by a thin continuous sheet of ice. The ice plate is modelled as an elastic shell, using the special Cosserat theory of hyperelastic shells (see Plotnikov $\&$ Toland 2011). We assume that the surface of the fluid coincides with the elastic shell, so that no gaps appear between them. Furthermore, the flow is assumed to be steady and irrotational. We introduce Cartesian coordinates $x^{*}$ and $y^{*}$, and align the $x^{*}$-axis along the bottom of the channel as $x^{*} \rightarrow \pm \infty$, with the $y^{*}$-axis directed vertically upwards through an obstacle. Gravitational acceleration $g$ acts in the negative $y^{*}$-direction.

We take $y^{*}=H+\eta^{*}\left(x^{*}\right)$ to define the deformation of the ice plate, and describe the bottom of the channel by $y^{*}=B^{*}\left(x^{*}\right)$. We seek hydraulic fall solutions so that the 


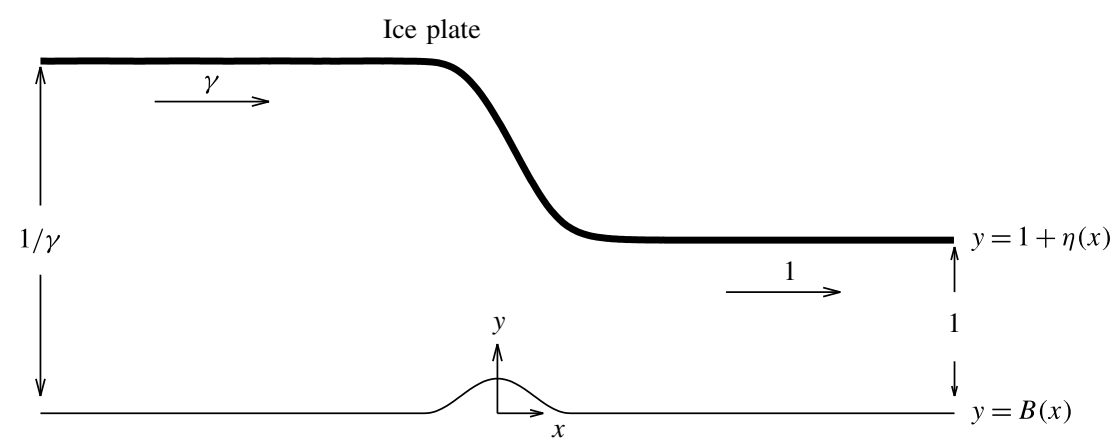

FIgURE 1. The dimensionless flow configuration over a single obstruction on the bottom of the channel.

depth of the fluid is greater upstream than downstream. The flow is thus assumed to be uniform in the far field as $x^{*} \rightarrow \pm \infty$, with constant depth $H$ and constant velocity $U$ downstream, and with constant depth $h(>H)$ and constant velocity $V(<U)$ upstream. The dimensionless downstream and upstream Froude numbers are then defined as

$$
F=\frac{U}{\sqrt{g H}} \quad \text { and } \quad F_{u p}=\frac{V}{\sqrt{g h}}
$$

respectively. We also introduce the parameter

$$
E_{b}=\frac{D}{\rho g H^{4}},
$$

where $D$ describes the flexural rigidity of the ice and $\rho$ is the density of the fluid.

We neglect the inertia and any stretching of the thin elastic ice sheet (see Squire et al. 1996 and Plotnikov \& Toland 2011 for more details) and next non-dimensionalise the problem using $U$ as unit velocity and $H$ as unit depth. Non-starred variables are thus now understood to be dimensionless, and the upstream dimensionless flow velocity is denoted by $\gamma$. The dimensionless flow configuration can be seen in figure 1. The depth of the fluid decreases downstream, and so the ice plate is seen to bend over the obstruction.

The velocity potential $\phi(x, y)$ is introduced, and the problem is then formulated as a system of nonlinear equations (see e.g. Lamb 1945) consisting of the Laplace equation in the fluid domain,

$$
\phi_{x x}+\phi_{y y}=0
$$

the kinematic conditions on the fluid surface and the channel bottom,

$$
\phi_{y}=\phi_{x} \eta_{x} \text { on } y=1+\eta(x) \text { and } \phi_{y}=\phi_{x} B_{x} \text { on } y=B(x),
$$

and the dynamic condition on the fluid surface (see e.g. Plotnikov \& Toland 2011; Guyenne \& Părău 2012),

$$
\frac{1}{2}\left(\phi_{x}^{2}+\phi_{y}^{2}\right)+\frac{1}{F^{2}}(y-1)-\frac{1}{2}+\frac{E_{b}}{F^{2}}\left(\kappa^{\prime \prime}+\frac{1}{2} \kappa^{3}\right)=0 \quad \text { on } y=1+\eta(x),
$$


having assumed that the ice sheet is not prestressed. Here, $\kappa$ describes the curvature of the fluid-ice interface and is given by $\kappa=\eta_{x x}\left(1+\eta_{x}^{2}\right)^{-3 / 2}$, and $E_{b} / F^{2}\left(\kappa^{\prime \prime}+\frac{1}{2} \kappa^{3}\right)$ describes the pressure from the ice plate on the fluid. The parameter $s$ is the arclength along the fluid surface, and the prime denotes differentiation with respect to $s$.

When computing hydraulic falls, the flow is required to be uniform in the far field, away from the submerged obstacles, so we impose the conditions

$$
\phi_{x} \rightarrow 1, y(x) \rightarrow 1 \quad \text { as } x \rightarrow \infty \quad \text { and } \quad \phi_{x} \rightarrow \gamma, y(x) \rightarrow \frac{1}{\gamma} \text { as } x \rightarrow-\infty
$$

Applying Bernoulli's equation in the far field as $x \rightarrow \pm \infty$, and using conservation of mass, one final equation is obtained (see Dias \& Vanden-Broeck 2004) to complete the system:

$$
\frac{1}{2}-\frac{1}{2} \gamma^{2}+\frac{1}{F^{2}}-\frac{1}{F^{2} \gamma}=0 .
$$

A relationship between the upstream and downstream Froude numbers in terms of the dimensionless upstream flow velocity $\gamma$ is given by (see Forbes 1988a)

$$
F_{u p}=F \gamma^{3 / 2}
$$

Following Belward \& Forbes (1993), Dias \& Vanden-Broeck (2004) and Page et al. (2014), the problem is now reformulated as a system of nonlinear integrodifferential equations, which can be solved for the unknown fluid surface $y=1+\eta(x)$ over arbitrarily shaped obstacles on the bottom of the channel. The fluid surface is first parametrised by writing $x=X(s)$ and $y=Y(s)$, so that the parametric equation

$$
X^{\prime 2}+Y^{\prime 2}=1
$$

is satisfied on the surface. The dynamic condition (2.5) is next rewritten as

$$
\frac{1}{2} \phi^{\prime 2}+\frac{1}{F^{2}}(Y(s)-1)-\frac{1}{2}+\frac{E_{b}}{F^{2}}\left(\kappa^{\prime \prime}+\frac{1}{2} \kappa^{3}\right)=0
$$

where $\kappa=Y^{\prime \prime} X^{\prime}-X^{\prime \prime} Y^{\prime}$ is the parametrised curvature of the fluid-ice interface.

Following Belward \& Forbes (1993), Cauchy's integral formula is applied to the function

$$
\frac{\mathrm{d} w}{\mathrm{~d} z}-\gamma=\phi_{x}-\gamma-\mathrm{i} \phi_{y}
$$

where $w(z)=\phi(x, y)+\mathrm{i} \psi(x, y)$ is the complex potential, $z$ is the complex variable $z=x+\mathrm{i} y$ and $\psi(x, y)$ is the stream function. The contour $\mathcal{C}$ is taken to consist of the fluid surface, the channel bottom and vertical lines joining them at $x= \pm \infty$. The two integral equations

$$
\begin{aligned}
\pi\left(\phi^{\prime}(s) X^{\prime}(s)-\gamma\right) & \\
= & -\int_{-\infty}^{\infty} \frac{\left(\phi^{\prime}(\sigma)-\gamma X^{\prime}(\sigma)\right)(Y(s)-Y(\sigma))-\gamma Y^{\prime}(\sigma)(X(\sigma)-X(s))}{(X(\sigma)-X(s))^{2}+(Y(\sigma)-Y(s))^{2}} \mathrm{~d} \sigma \\
& +\int_{-\infty}^{\infty} \frac{\left(\hat{u}(\sigma)\left(1+B_{x}(\sigma)^{2}\right)-\gamma\right)(Y(s)-B(\sigma))-\gamma B_{x}(\sigma)(\sigma-X(s))}{(\sigma-X(s))^{2}+(B(\sigma)-Y(s))^{2}} \mathrm{~d} \sigma
\end{aligned}
$$


and

$$
\begin{aligned}
\pi(\hat{u}(x)-\gamma)= & -\int_{-\infty}^{\infty} \frac{\left(\phi^{\prime}(\sigma)-\gamma X^{\prime}(\sigma)\right)(B(x)-Y(\sigma))-\gamma Y^{\prime}(\sigma)(X(\sigma)-x)}{(X(\sigma)-x)^{2}+(Y(\sigma)-B(x))^{2}} \mathrm{~d} \sigma \\
& +\int_{-\infty}^{\infty} \frac{(-B(\sigma)+B(x))\left(\hat{u}(\sigma)\left(1+B_{x}(\sigma)^{2}\right)-\gamma\right)-\gamma B_{x}(\sigma)(\sigma-x)}{(\sigma-x)^{2}+(B(\sigma)-B(x))^{2}} \mathrm{~d} \sigma
\end{aligned}
$$

are derived, where $\sigma$ represents the value of the arclength at the varying point $z(\sigma)=$ $x(\sigma)+\mathrm{i} y(\sigma)$ on the contour $\mathcal{C}$, and the evaluation points $s$ and $x$ are placed on the free surface and the channel bottom, respectively. The horizontal velocity of the fluid on the channel bottom is defined by $u(\sigma, B(\sigma))=\hat{u}(\sigma)$.

The two integral equations (2.12) and (2.13), together with the parametrised dynamic condition (2.10) and the parametric equation (2.9), form the system of integrodifferential equations to be solved, iteratively via Newton's method, for the unknown variables $Y^{\prime}(s), X^{\prime}(s)$ and $\phi^{\prime}(s)$ at the fluid-ice interface and $\hat{u}(x)$ on the channel bottom.

\section{Results}

Following Dias \& Vanden-Broeck (2004) and Page et al. (2014), a cosine-squared profile of the form

$$
B(x)= \begin{cases}2 A_{1} \cos ^{2}\left(\frac{\pi\left(x+x_{1}\right)}{2 L_{1}}\right), & -L_{1}<x+x_{1}<L_{1}, \\ 2 A_{2} \cos ^{2}\left(\frac{\pi(x)}{2 L_{2}}\right), & -L_{2}<x<L_{2}, \\ 0, & \text { otherwise }\end{cases}
$$

is used as the channel bottom profile. The heights and half-lengths of the submerged obstructions are thus defined by $2 A_{i}$ and $L_{i}(i=1,2)$, respectively. The separation constant $x_{1}$ describes the central position of the additional obstruction. In the case of just a single submerged obstruction, $A_{1}$ is taken to be zero.

\subsection{Linear theory}

In order to explain some of the results in this section, we briefly review the linearised theory. By substituting a linear periodic wave function with wavenumber $k$ into the linearisation of the governing equations downstream, i.e. (2.3)-(2.5), we obtain the downstream linear dispersion relation

$$
F^{2}=\left(\frac{1}{K}+E_{b} K^{3}\right) \tanh (K) .
$$

Here $K=k H$ is the dimensionless wavenumber of the waves.

Similarly, upon substituting the linear periodic wave function with wavenumber $k$ into the linearisation of the governing equations upstream and then non-dimensionalising with respect to the downstream fluid depth $H$, the upstream linear dispersion relation is obtained:

$$
F_{u p}^{2}=\left(\frac{\gamma}{K}+E_{b} K^{3} \gamma\right) \tanh \left(\frac{K}{\gamma}\right) .
$$



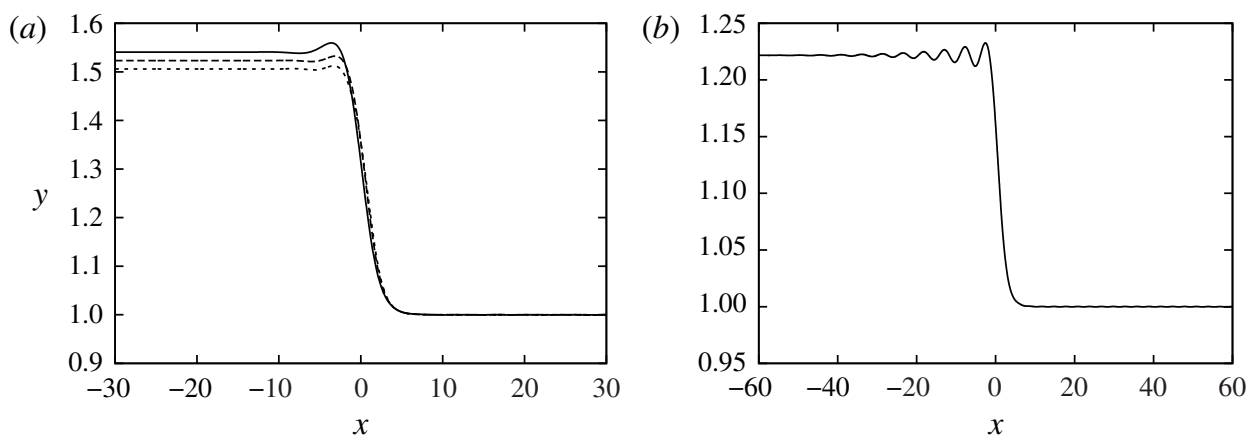

FIGURE 2. Hydraulic fall profiles over a single submerged obstruction of: $(a)$ height $2 A_{2}=0.1$ and length $2 L_{2}=6 ;(b)$ height $2 A_{2}=0.02$ and length $2 L_{2}=6.4$. An elevation appears immediately before the fall in $(a)$ for the sets of parameter values $E_{b}=0.5, F=$ $1.367, F_{u p}=0.715$ (solid curve), $E_{b}=0.2, F=1.356, F_{u p}=0.721$ (dotted curve) and $E_{b}=0.1, F=1.345, F_{u p}=0.728$ (dashed curve). A train of decaying waves upstream before the fall is shown in $(b)$ for the parameters $E_{b}=0.1, F=1.159$ and $F_{u p}=0.858$. In each case, the Froude number was found as part of the solution.

An important property of the flexural-gravity linear dispersion relation is that there is always a point at which the phase velocity is equal to the group velocity (see Squire et al. 1996; Guyenne \& Părău 2012). Therefore, there exists a minimum Froude number $F_{u p_{\text {min }}}$, with corresponding wavenumber $k=k_{\text {min }}$. Near this minimum, linear theory fails and nonlinear theories are required.

\subsection{Fully nonlinear results}

Hydraulic fall solutions with subcritical flow upstream $\left(F_{u p}<1\right)$ and supercritical flow downstream $(F>1)$ are sought using the numerical scheme described in $\S 2$ (see also Page et al. 2014).

To ensure the accuracy of the results, the same solution was obtained on meshes of different densities and sizes. The number of points on the fluid-ice interface was varied in the range $N=401, \ldots, 2401$, and the number on the channel bottom in the range $M=201, \ldots, 801$. The mesh spacing was similarly taken to be $e=0.1,0.05,0.025$ on the free-surface and $h=0.2,0.1,0.05$ on the channel bottom. By fixing the domain length, we were therefore able to determine that our solutions are independent of the mesh, provided that the mesh spacing is small enough. Where appropriate, solutions were also computed on meshes of different lengths but with the same mesh density, to ensure that our solutions are not affected by truncation effects.

We take $A_{1}=0$ and $A_{2}>0$, and fix the value of $L_{2}$ so that the function $y=B(x)$ describes a uniform channel bottom with just a single obstruction. Typical hydraulic fall profiles with $E_{b}=0.5, E_{b}=0.2$ and $E_{b}=0.1$ over an obstruction characterised by $A_{2}=0.05$ and $L_{2}=3$ are shown in figure $2(a)$. Immediately before the hydraulic fall, there exists a slight elevation in the ice plate deformation. A similar phenomenon was observed for weak gravity-capillary hydraulic falls (i.e. with $\tau<\frac{1}{3}$, where $\tau$ is the Bond number defined by $\tau=\sigma /\left(\rho g H^{2}\right)$ with $\sigma$ being the surface tension coefficient) found in the neighbourhood of the minimum of the upstream gravity-capillary linear dispersion relation (see Guayjarernpanishk \& Asavanant 2012; Page et al. 2014).

Small-amplitude spurious periodic waves are sometimes found downstream of the hydraulic fall, where the flow is supercritical. In this region, the downstream Froude 


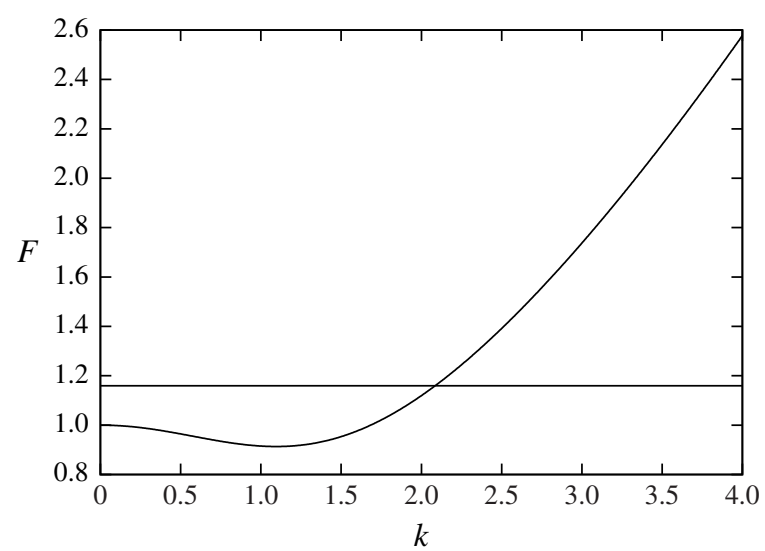

FIgURE 3. Downstream dispersion relation of figure $2(b)$. The horizontal line corresponds to the downstream value of the Froude number found in figure $2(b)$.

number intersects the linear dispersion curve (see figure 3 for the linear dispersion relation corresponding to figure $2 b$ ), and so (short) flexural waves are indeed predicted by the linear theory ahead of a forcing. There is no obstacle to generate these waves in this downstream region, but the downstream truncation can act as a form of artificial forcing, ahead of which waves can form. However, by manipulating the far-field conditions downstream, it is possible to reduce the amplitude of the spurious waves so much so that we obtain approximately zero-amplitude waves downstream, and thus physically relevant solutions.

By decreasing the value of the parameter $E_{b}$, the upstream Froude number increases towards the minimum of the upstream linear dispersion relation, and the small elevation becomes part of a train of decaying waves before the fall. A similar result has been seen in the critical solutions for the gravity-capillary case. However, the train of waves found here in the flexural-gravity cases appear to be more pronounced and extend further upstream. In order to minimise any truncation problems, we use a long domain, truncating at $x= \pm 60$. Figure $2(b)$ shows such a train of waves immediately before a fall for the parameters $E_{b}=0.1$ and $F=1.159$. The upstream Froude number is found to be $F_{u p}=0.858$, which is very close to the minimum of the upstream linear dispersion relation, $F_{u p_{\text {min }}} \approx 0.86$, with corresponding wavenumber $k_{\text {min }} \approx 1.2$.

In the experiments at Lake Saroma, Japan (see Takizawa 1985, 1988), the water depth was $6.8 \mathrm{~m}$, which would correspond to $E_{b}=0.01$ here. In the Lake Diefenbaker (Canada) experiments, the water depth was $35 \mathrm{~m}$ with the parameter $E_{b}=0.03$. The values $E_{b}=0.5,0.2$ and 0.1 for the solutions in figure 2(a) would correspond, respectively, to physical water depths of 2.61, 3.29 and $3.91 \mathrm{~m}$ in Lake Saroma, and 17.51, 22.02 and $26.19 \mathrm{~m}$ in Lake Diefenbaker. Decreasing the value of the parameter $E_{b}$ corresponds to increasing the depth of the water downstream.

The solution branch in the $F-E_{b}$ plane, for $0.08<E_{b}<5$ and with an underlying submerged obstacle characterised by $A_{2}=0.05$ and $L_{2}=3$, is plotted in figure $4(a)$. One finds that the Froude number increases with $E_{b}$ up to some critical value, $E_{b}^{*}$. Conversely, as $E_{b}$ decreases (towards $E_{b}=0.08$ ), we approach the minimum of the upstream linear dispersion relation, and small-amplitude numerical oscillations begin to appear on the branch. A longer domain, with more mesh points on the surface, 

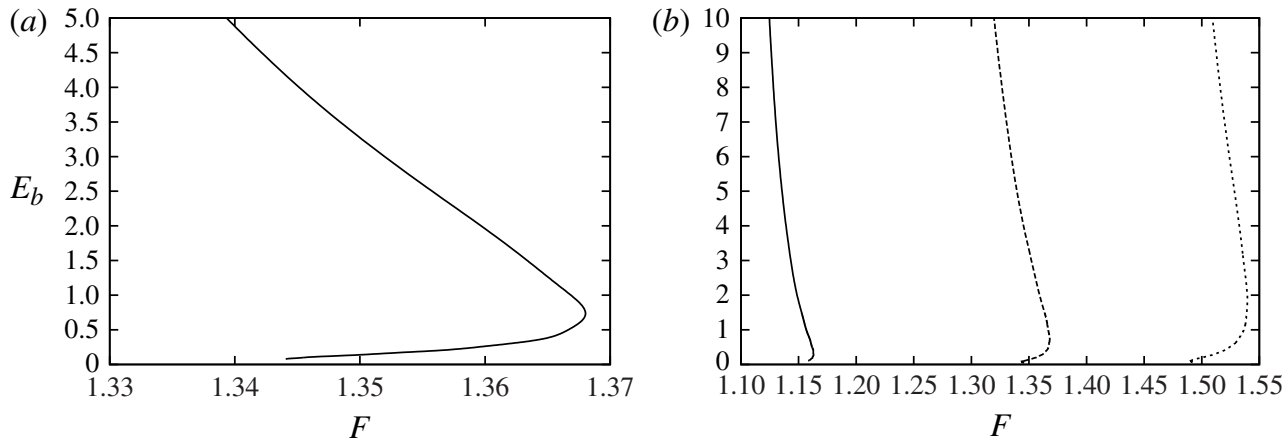

FIgURE 4. Solution branches in the $F-E_{b}$ plane downstream, for a single submerged obstruction characterised by: (a) $A_{2}=0.05, L_{2}=3$; (b) $A_{2}=0.01, L_{2}=3.2$ (solid line), $A_{2}=0.05, L_{2}=3$ (dashed line) and $A_{2}=0.1, L_{2}=3.2$ (dotted line).

is required to produce accurate solutions. Further turning points may exist on the branch as one continues to reduce $E_{b}$. Due to computational limitations, we therefore truncated the lower end of the branch at $E_{b}=0.08$, before getting too close to the minimum.

At $E_{b}^{*}$, a turning point exists in the $F-E_{b}$ plane, after which the Froude number decreases monotonically as $E_{b}$ is increased. The existence of this turning point means that for a critical range of Froude numbers, there exist two solutions over the same underlying obstruction, with the same value of the Froude number but different values of $E_{b}$. Two such solutions are shown in figure $5(a)$ for $F=1.36$; the two values of $E_{b}$ are 1.5 and 0.33 . It should be noted that for such solutions, as the density $\rho$, the gravitational acceleration $g$ and the flexural rigidity of the ice $D$ are fixed in both cases, having different values of $E_{b}$ corresponds to having different values of $H$ and thus different downstream fluid depths. Further, as the Froude number is also fixed in both solutions, this implies that the downstream fluid speed in the two solutions differs. The phenomenon discussed here is therefore not bistability in the traditional sense.

Figure $4(b)$ shows the solution branches in the $F-E_{b}$ plane for three different underlying obstacles. The downstream Froude number and the critical value $E_{b}^{*}$ at which the turning point exists increase with obstacle size. Similar behaviour was found for the $\tau-F$ branches in the gravity-capillary case; see figure 6 of Page et al. (2014). However, the critical range of Froude numbers for which there exist two solutions with the same value of the Froude number but different values of $\tau$ is larger than the comparable critical range in the flexural-gravity case for two different values of $E_{b}$.

Increasing the height or width of the obstacle decreases the upstream Froude number, so that with $E_{b}$ fixed, the difference between $F_{u p}$ and $F_{u p_{\min }}$ increases. We were therefore able to reduce $E_{b}$ further, before $F_{u p}$ intersected the upstream linear dispersion relation, by increasing the size of the obstacle. A solution with $E_{b}=0.02$ over an obstacle of height $2 A_{2}=0.35$ and width $2 L_{2}=35$ is plotted as the solid curves in figure $5(b)$. A solution over a smaller obstacle (with a larger value of $E_{b}$ ) is represented by the dashed curves. The slope of the fall clearly decreases for solutions over a wider or larger obstacle. Similarly, the upstream depth of the fluid increases with the height of the obstacle.

In the absence of a thin ice sheet covering the fluid, placing an additional obstruction upstream of the hydraulic fall in the pure gravity case has the effect 

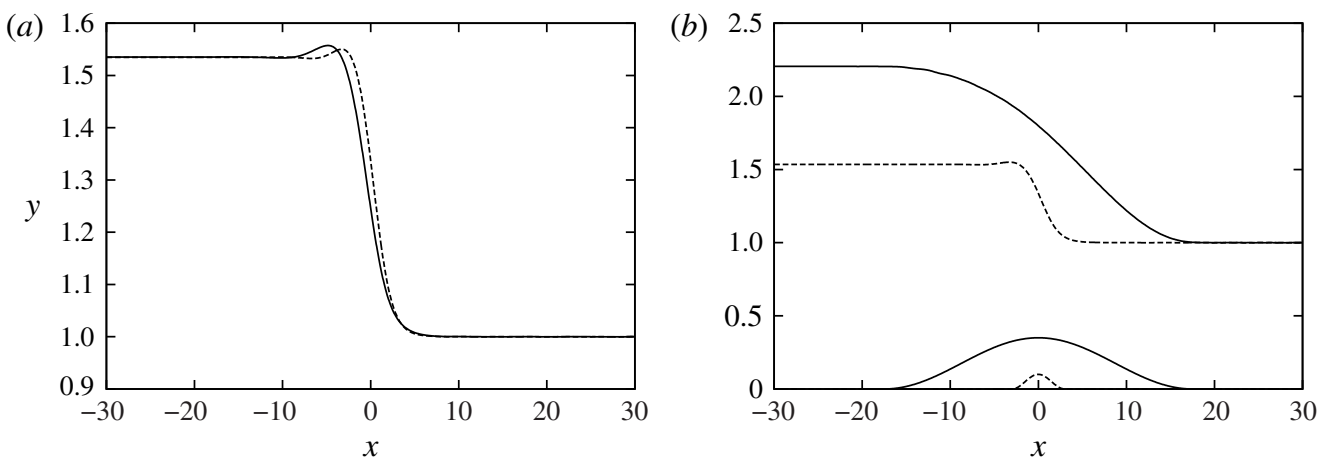

FIgURE 5. (a) Hydraulic fall solutions over a submerged obstacle of height $2 A_{2}=0.1$ and width $2 L_{2}=6$ : the solid curve is the solution with $E_{b}=1.5$ and the dashed curve is the solution with $E_{b}=0.33$; the Froude number in both cases is found to be $F=1.36$. (b) Hydraulic fall solutions over a submerged obstacle: the solid curve is the solution over an obstacle of height $2 A_{2}=0.35$ and width $2 L_{2}=35$, with $F=1.74$ and $E_{b}=0.02$; the dashed curve is the same as the dashed solution in $(a)$; the obstacles in both cases are also shown.

of producing a train of trapped waves between the two obstacles before the hydraulic fall (see Dias \& Vanden-Broeck (2004) for numerical solutions and Pratt (1984) for experimental results). In the gravity-capillary problem, however, unless the surface tension is very small so that the upstream Froude number intersects the upstream gravity-capillary linear dispersion relation, the additional obstacle must be placed downstream of the hydraulic fall in order to obtain a train of trapped waves between the two obstacles (Page et al. 2014).

We place the additional obstruction downstream of the hydraulic fall to obtain trapped wave solutions. A typical profile with the additional obstacle centred at $x=20$ is shown in figure $6(a)$. A train of waves exists between the two underlying obstructions, with a higher-amplitude elevation wave occurring over the second obstruction. In this downstream region, the flow is supercritical and pure gravity flows without trapped waves have been considered by Belward (1999). A small elevation appears upstream immediately before the fall, over the first obstruction.

The wavelength of these waves can be inferred from the linear theory, with the downstream Froude number intersecting the downstream linear dispersion relation at wavenumber $K \approx 1.573$. Both the upstream and downstream dispersion relations are shown in figure 7 . A wavetrain of waves of wavelength $\lambda=2 \pi / 1.573 \approx 4.002$ (solid line) is therefore expected. The waves found in figure $6(a)$ are of wavelength $\lambda \approx$ $12.83-8.85=3.98$, which is very close to the wavelength predicted by the linear theory.

Reducing the amplitude of the additional obstacle or reducing the parameter $E_{b}$ reduces the amplitude of the trapped waves.

The solution branch in the $E_{b}-F$ plane (with $E_{b} \leqslant 15$ ) is shown in figure $6(b)$ for the channel bottom configuration characterised by $A_{2}=0.1, A_{1}=0.05$ and $L_{1}=$ $L_{2}=3.2$. Multiple turning points are found on the branch, showing that for a critical range of Froude numbers, the trapped wave solution for a given underlying channel bottom configuration is not unique. For example, for $F=1.51$, figure 8 shows five different solutions for different values of $E_{b}$, namely $E_{b}=0.28,0.43,0.54,1.17$ and 5.51 , corresponding to water depths of $3.02,2.71,2.56,2.11$ and $1.43 \mathrm{~m}$, respectively, 

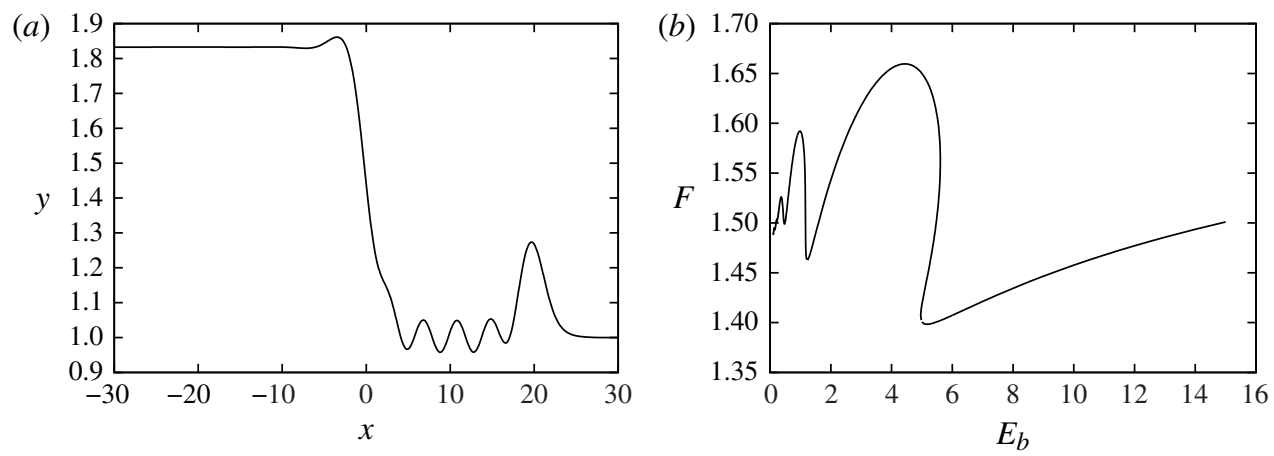

FIgURE 6. (a) Hydraulic fall profile over a submerged obstacle of height $2 A_{2}=0.2$ and width $2 L_{2}=6.4$, with an additional obstacle, characterised by $2 A_{1}=0.16$ and $2 L_{1}=6.4$ with $x_{1}=-20$, downstream: a train of trapped waves exists between the obstacles; the Froude number $F=1.54$ is found as part of the solution, and $E_{b}=0.5$ is given. (b) The trapped wave solution branch in the $E_{b}-F$ plane for flow past two fixed submerged obstructions characterised by $A_{2}=0.1, A_{1}=0.05$ and $L_{1}=L_{2}=3.2$ with $x_{1}=-15$.

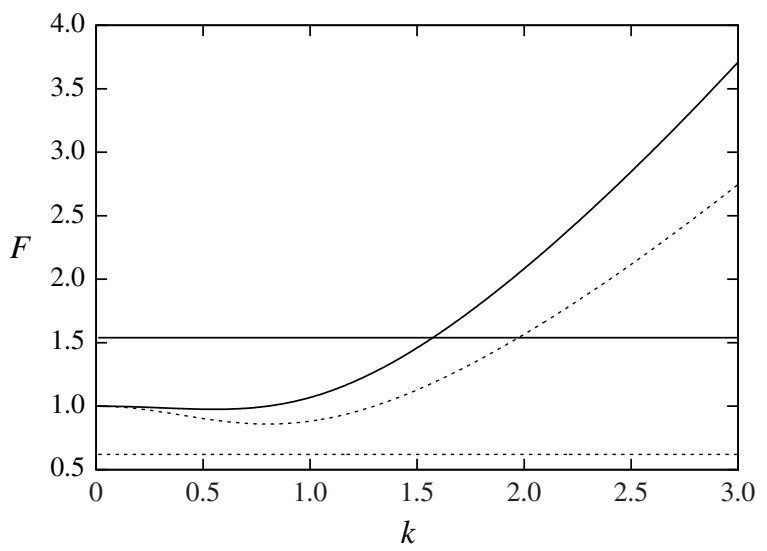

FIGURE 7. Linear dispersion relations for $E_{b}=0.5$. The solid curve gives the downstream linear dispersion relation, and the solid line at constant $F$ gives the downstream value of the Froude number for a channel bottom configuration classified by $A_{2}=0.1, A_{1}=$ $0.08, L_{1}=L_{2}=3.2$ and $x_{1}=-20$. The dashed curve gives the upstream linear dispersion relation, and the dashed line the upstream Froude number for the same configuration.

for the physical parameters used in the experiments at Lake Saroma in Japan, and to water depths of $20.25,18.19,17.18,14.16$ and $9.61 \mathrm{~m}$ for the physical parameters in the experiments at Lake Diefenbaker in Canada. The amplitude of the trapped waves can be seen to increase with $E_{b}$. We were able to continue following the solution branch in figure $6(b)$ for much greater values of $E_{b}$, with $F$ increasing monotonically with $E_{b}$, corresponding to smaller and smaller channel depths.

We calculated solutions for obstacles of different heights and widths from those shown in figure 6 , and found similar behaviours to the results described here. A solution with $A_{1}>A_{2}$ is shown in figure $9(a)$. The height of the elevation over the obstacle downstream of the hydraulic fall may increase so much that it is of the same height as the upstream flow. When $A_{1}<0$, similar trapped wave solutions can 

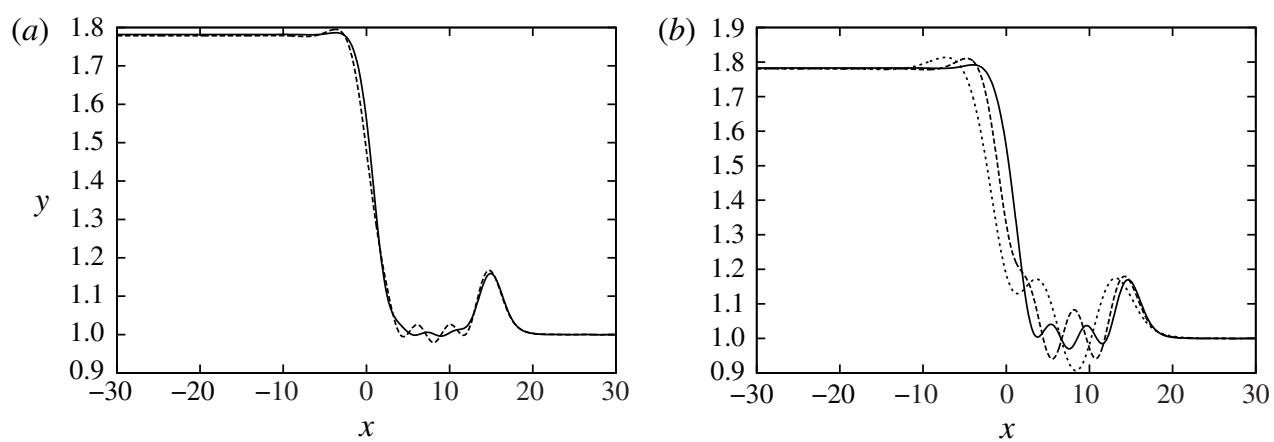

FIgURE 8. Trapped wave solutions for flow past two fixed submerged obstructions characterised by $A_{2}=0.1, A_{1}=0.05$ and $L_{1}=L_{2}=3.2$ with $x_{1}=-15$, for different values of the parameter $E_{b}$ : (a) $E_{b}=0.28$ (solid curve) and $E_{b}=0.43$ (dashed curve); (b) $E_{b}=0.54$ (solid curve), $E_{b}=1.17$ (dashed curve) and $E_{b}=5.51$ (dotted curve). The Froude number in each case is found to be $F=1.51$.
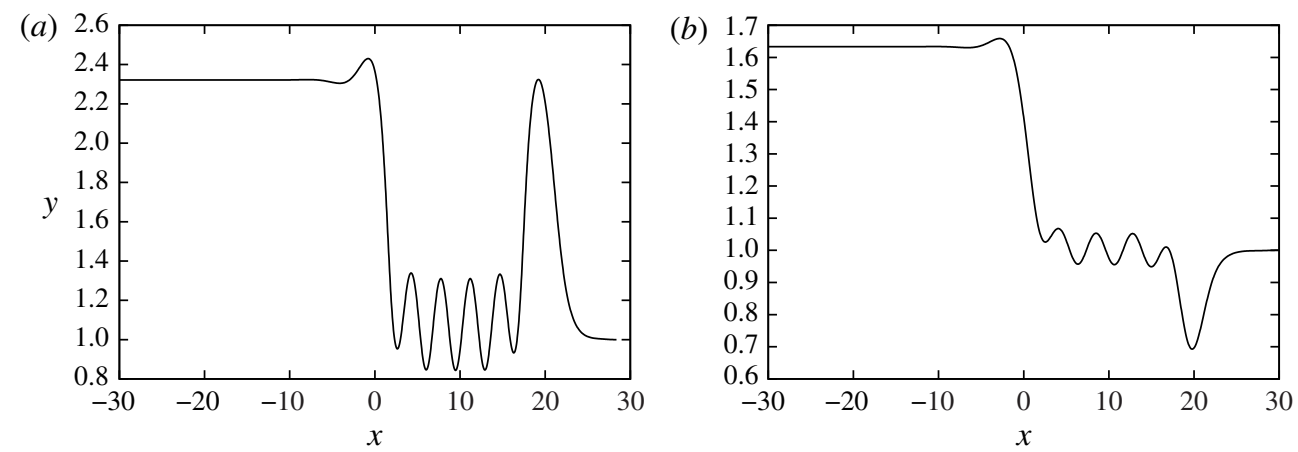

FIGURE 9. Hydraulic fall profiles over a submerged obstacle of width $2 L_{2}=6.4$, with an additional obstacle of width $2 L_{1}=6.4$ at $x_{1}=-20$ downstream: (a) submerged obstacle of height $2 A_{2}=0.2$ and downstream obstacle of height $2 A_{1}=0.6 ;(b)$ submerged obstacle of height $2 A_{2}=0.1$ and downstream obstacle of height $2 A_{1}=-0.2$. A train of waves exists between the obstacles. The Froude numbers, $F=1.8$ for $(a)$ and $F=1.42$ for $(b)$, are found as part of the solution, and $E_{b}=0.5$ is given.

be found (see figure $9 b$ ), but here a depression wave appears over the additional obstruction downstream.

In the absence of a sheet of ice, generalised hydraulic falls which have a train of non-decaying waves upstream of the fall may be obtained in both the pure gravity case (Dias \& Vanden-Broeck 2002) and the gravity-capillary (Page et al. 2014) case. These solutions lack physical relevance in a flow configuration involving just a single obstruction, however, due to violating the radiation condition which requires that no energy comes from infinity (and thus no gravity waves appear upstream). In the hydroelastic problem, generalised hydraulic fall solutions are also found to exist for small $E_{b}$, where the upstream Froude number intersects the upstream linear dispersion relation twice. The resonance between the two modes is similar to the resonance in the gravity-capillary case (see, for example, Wilton 1915; Vanden-Broeck 2002), and thus waves of two different wavelengths travelling at the same speed can appear on 
(a)

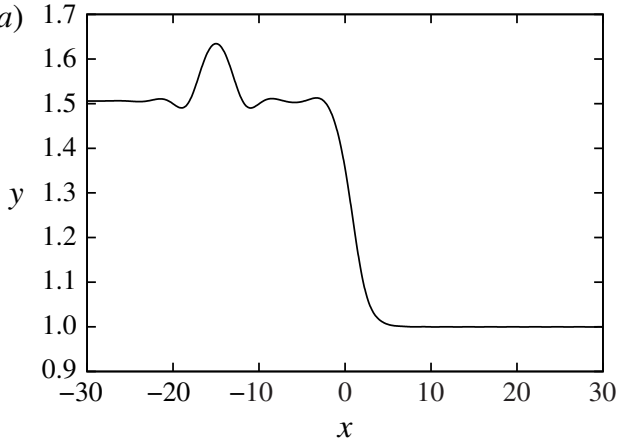

(b)

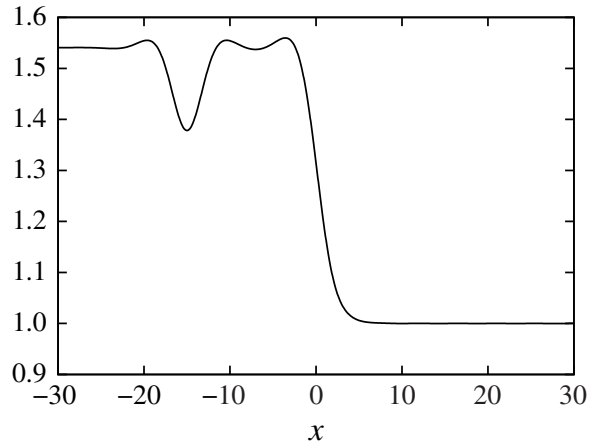

FIGURE 10. Hydraulic fall profiles over a submerged obstruction of height $2 A_{2}=0.1$ and width $2 L_{2}=6$, with an additional obstacle upstream at $x=-15$, characterised by: $(a) A_{1}=$ -0.1 and $L_{1}=3 ;(b) A_{1}=0.05$ and $L_{1}=3$. The Froude numbers $F=1.34$ and $F=1.37$, respectively, are found as part of the solution, and the parameters $E_{b}=0.1$ and $E_{b}=0.5$ are given.

the upstream part of the solution (see Vanden-Broeck \& Părău 2011). However, the accurate computation of these waves is difficult, and we do not present results in this region due to the computational limitations.

When the additional obstacle is placed upstream of the hydraulic fall with $F_{u p}$ less than the minimum of the upstream linear dispersion relation, a forced solitary-type wave with small decaying oscillations in its tail is obtained over the obstruction. It is worth noting that in shallow water, for $F \approx 1$, a fifth-order $\mathrm{KdV}$ equation was derived by Guyenne \& Părău (2014) and Xia \& Shen (2002). It is well known that this equation admits solitary waves with decaying oscillations, as solutions for $F_{u p}<F_{u p_{\text {min }}}$ (see Grimshaw, Malomed \& Benilov 1994). Forced waves with decaying oscillations for a forced fifth-order $\mathrm{KdV}$ equation have also been computed by Cho \& Akylas (2009). We obtain a depression wave if $A_{1}>0$ and an elevation wave if $A_{1}<0$. Example solution profiles are shown in figure 10. Decreasing $E_{b}$ so that the upstream Froude number approaches the minimum of the upstream linear dispersion relation increases the number of decaying oscillations in the tails of these forced solitary-type waves.

\section{Discussion}

Fully nonlinear hydraulic fall solutions under an ice plate modelled by a thin elastic shell have been presented and discussed. A train of decaying waves immediately before the fall is observed. When an additional obstruction is placed downstream of the hydraulic fall, a train of waves trapped between the obstacles has been found. It is shown that such solutions are not unique. When the additional obstacle is placed upstream, forced solitary-type waves with small decaying oscillations in their tails exist.

The values of $E_{b}$ used in our results are relatively large, and so the results presented in this paper correspond to shallow-water applications (e.g. lakes and fjords) rather than deeper oceanographic situations. Typical physical values for the parameters used in the Lake Saroma experiments (see Takizawa 1985, 1988) are $h=0.17 \mathrm{~m}, \rho=$ $1026 \mathrm{~kg} \mathrm{~m}^{-3}, g=9.8 \mathrm{~m} \mathrm{~s}^{-2}, E=5.1 \times 10^{5} \mathrm{~N} \mathrm{~m} \mathrm{~s}^{-2}$ and $H=6.8 \mathrm{~m}$, where $h$ is the thickness of the ice, $E$ is the Young's modulus and $H$ is the water depth. The 
flexural rigidity of the ice is given by $D=E h^{3} / 12\left(1-v^{2}\right) \approx 2.34 \times 10^{5} \mathrm{~N}$ m where $v$ is the Poisson ratio (see Părău \& Dias 2002). This corresponds to $E_{b}=0.01$. In the Lake Diefenbaker experiments (see Eyre 1977) the physical parameters can be taken to be $h=0.73 \mathrm{~m}, \rho=1026 \mathrm{~kg} \mathrm{~m}^{-3}, E=13 \times 10^{9} \mathrm{~N} \mathrm{~m} \mathrm{~s}^{-2}$ and $H=35 \mathrm{~m}$. This gives an approximate flexural rigidity of the ice of $D \approx 4.73 \times 10^{8} \mathrm{~N} \mathrm{~m}$ and thus $E_{b}=0.03$. Here, we have computed solutions for $E_{b}$ from 0.02 to 5 (see figure $4(a)$ ). Such solutions would thus be physically relevant for Lake Saroma between depths 5.84 and $1.47 \mathrm{~m}$, and for Lake Diefenbaker between depths 39.16 and $9.85 \mathrm{~m}$. One can see that the mean depth of Lake Diefenbaker is included within this range.

Computing solutions for small values of $E_{b}$ is difficult, as they lie in the neighbourhood of the minimum of the upstream linear dispersion relation. Reducing $E_{b}$, whilst keeping the size of the obstacle fixed, increases the upstream Froude number, so that it approaches $F_{u p_{\text {min }}}$. When the Froude number intersects the upstream linear dispersion relation, a resonance occurs, and thus waves of two different wavelengths are expected to travel at the same speed on the upstream part of the solution. Therefore, solutions corresponding to greater depths in Lake Saroma and Lake Diefenbaker over the fixed obstacle are expected to take the form of, for example, Wilton ripples upstream of the fall (Vanden-Broeck 2002), whilst being uniform downstream.

If, however, one increases the size of the obstacle, this decreases $F_{u p}$ so that $E_{b}$ may be reduced further before $F_{u p}$ intersects the linear dispersion relation. For a large obstacle, the hydraulic falls found at greater depths in Lake Saroma and Lake Diefenbaker may therefore be uniform both upstream and downstream.

It should be noted that in some cases, for very-large-amplitude solutions which correspond to large obstacles, the model described here can become unphysical. For realistic solutions, the strain of the ice plate needs to be less than the yield strain of ice (see Brocklehurst, Korobkin \& Părău 2010). Otherwise, at these high strains, it becomes unrealistic to model the ice plate as a thin elastic shell, as the behaviour of the plate becomes plastic and so one would expect the ice to fracture or break. The strain of the ice plate may be calculated by

$$
\epsilon=\frac{h}{2} \kappa^{*} \approx \frac{h}{2} \eta_{x^{*} x^{*}}^{*},
$$

where $h$ is the thickness of the ice and $\kappa^{*}$ is the dimensional curvature of the ice plate (see e.g. Ugural 1981, Brocklehurst et al. 2010 and Squire 1993 for more details). The strain is thus proportional to the curvature of the surface. For short waves, the increase of the curvature in the deformation of the ice plate therefore causes greater strain in the plate. The maximum strain in the solutions presented in this paper occurs at the beginning and the end of the hydraulic falls. We calculated it to be within the region $1 \times 10^{-3}$ to $1 \times 10^{-5}$, depending on the height and width of the obstacle. As the amplitude of the obstacle is reduced or the width is increased, the maximum strain in the ice plate is found to decrease. Failure of the ice occurs as a result of cracks propagating through the ice. In the ocean, Goodman, Wadhams \& Squire (1980) calculated that a crack would propagate if the strain reached the critical value $\epsilon_{c r}=4.3 \times 10^{-5}$. In pure ice, however, the yield strain is higher as salt does not creep into the cracks, so $\epsilon_{c r}=2.14 \times 10^{-4}$ in this case (see Goodman et al. 1980). The solutions presented here with the steepest hydraulic falls have the largest values of the strain, of the order of $10^{-3}$, and would thus be expected to fracture. However, physically realistic solutions were also presented in this paper, where the strain is below $\epsilon_{c r}$. 


\section{Acknowledgements}

The research presented in this paper was carried out on the High Performance Computing Cluster supported by the Research and Specialist Computing Support service at the University of East Anglia. E.I.P. was supported by the EPSRC under grant EP/J019305/1. Both authors also wish to thank the anonymous referees for their helpful and constructive comments.

\section{REFERENCES}

Antman, S. S. 2005 Nonlinear Problems of Elasticity. 2nd edn. Springer.

Ashton, G. D. 1986 River and Lake Ice Engineering. Water Resources Publications.

BELWARD, S. R. 1999 Fully nonlinear flow over successive obstacles: hydraulic fall and supercritical flows. J. Austral. Math. Soc. B 40, 447-458.

Belward, S. R. \& Forbes, L. K. 1993 Fully non-linear two-layer flow over arbitrary topography. J. Engng Maths 27, 419-432.

Brocklehurst, P., Korobkin, A. A. \& PăRĂU, E. I. 2010 Interaction of hydro-elastic waves with a vertical wall. J. Engng Maths 68 (3-4), 215-231.

Cho, Y. \& Akylas, T. R. 2009 Forced waves near resonance at a phase-speed minimum. Stud. Appl. Maths 123 (1), 1-15.

Dias, F. \& VAnden-Broeck, J.-M. 2002 Generalised critical free-surface flows. J. Engng Maths 42 (3-4), 291-301.

Dias, F. \& VAnden-Broeck, J.-M. 2004 Trapped waves between submerged obstacles. J. Fluid Mech. 509, 93-102.

EYRE, D. 1977 The flexural motions of a floating ice sheet induced by moving vehicles. J. Glaciol. 19 (81), 555-570.

Forbes, L. K. 1986 Surface waves of large amplitude beneath an elastic sheet. Part 1. High-order series solution. J. Fluid Mech. 169, 409-428.

Forbes, L. K. 1988 a Critical free-surface flow over a semi-circular obstruction. J. Engng Maths 22, 3-13.

Forbes, L. K. $1988 b$ Surface waves of large amplitude beneath an elastic sheet. Part 2. Galerkin solution. J. Fluid Mech. 188, 491-508.

Goodman, D. J., Wadhams, P. \& SQuire, V. A. 1980 The flexural response of a tabular ice island to ocean swell. Ann. Glaciol. 1, 23-27.

Grimshaw, R., Malomed, B. \& Benilov, E. 1994 Solitary waves with damped oscillatory tails: an analysis of the fifth-order Korteweg-de Vries equation. Physica D 77 (4), 473-485.

GuAYJARERnPANishK, P. \& AsaVAnANT, J. 2012 Free-surface flows over an obstacle: problem revisited. In Modelling, Simulation and Optimization of Complex Processes (ed. H. G. Bock, X. P. Hoang, R. Rannacher \& J. P. Schlöder), pp. 139-151. Springer.

GuYenne, P. \& PĂRĂU, E. I. 2012 Computations of fully nonlinear hydroelastic solitary waves on deep water. J. Fluid Mech. 713, 307-329.

Guyenne, P. \& PĂRĂU, E. I. 2014 Finite depth effects on solitary waves in a floating ice sheet. J. Fluids Struct. (submitted).

Korobkin, A., PĂRăU, E. I. \& VAnden-Broeck, J.-M. 2011 The mathematical challenges and modelling of hydroelasticity. Phil. Trans. R. Soc. A 369 (1947), 2803-2812.

LAMB, H. 1945 Hydrodynamics. 6 edn. Dover Publications.

Milewski, P. A., VAnden-Broeck, J.-M. \& WAng, Z. 2011 Hydroelastic solitary waves in deep water. J. Fluid Mech. 679, 628-640.

Page, C., Grandison, S. \& PĂRĂU, E. I. 2014 The influence of surface tension upon trapped waves and hydraulic falls. Eur. J. Mech. B/Fluids 43, 191-201.

PĂRĂU, E. I. \& DIAS, F. 2002 Nonlinear effects in the response of a floating ice plate to a moving load. J. Fluid Mech. 460, 281-305.

Plotnikov, P. I. \& Toland, J. F. 2011 Modelling nonlinear hydroelastic waves. Phil. Trans. R. Soc. A 369 (1947), 2942-2956. 
Pratt, L. J. 1984 On nonlinear flow with multiple obstructions. J. Atmos. Sci. 41, 1214-1225.

SQuire, V. A. 1993 The breakup of shore fast sea ice. Cold Reg. Sci. Technol. 21 (3), 211-218.

SQuire, V. A. 2011 Past, present and impendent hydroelastic challenges in the polar and subpolar seas. Phil. Trans. R. Soc. A 369 (1947), 2813-2831.

Squire, V. A., Hosking, R. J., Kerr, A. D. \& Langhorne, P. J. 1996 Moving Loads on Ice Plates. Springer.

TAKizawA, T. 1985 Deflection of a floating sea ice sheet induced by a moving load. Cold Reg. Sci. Technol. 11 (2), 171-180.

TAKIZAWA, T. 1988 Response of a floating sea ice sheet to a steadily moving load. J. Geophys. Res. 93 (C5), 5100-5112.

Ugural, A. 1981 Stresses in Plates and Shells. McGraw Hill.

VANDEN-BROECK, J.-M. 2002 Wilton ripples generated by a moving pressure distribution. J. Fluid Mech. 451, 193-201.

VANDEN-Broeck, J.-M. \& PĂRĂU, E. I. 2011 Two-dimensional generalized solitary waves and periodic waves under an ice sheet. Phil. Trans. R. Soc. A 369 (1947), 2957-2972.

Wang, Z., VAnden-Broeck, J.-M. \& Milewski, P. A. 2013 Two-dimensional flexural-gravity waves of finite amplitude in deep water. IMA J. Appl. Maths 78 (4), 750-761.

Wilton, J. R. 1915 On ripples. Phil. Mag. Ser. 629 (173), 688-700.

XIA, X.\& SHEN, H. T. 2002 Nonlinear interaction of ice cover with shallow water waves in channels. J. Fluid Mech. 467, 259-268. 